УДК 665.658 .6

\title{
КАТАЛИТИЧЕСКИЙ ПАРОВОЙ КРЕКИНГ ГУДРОНА В ПРИСУТСТВИИ ДИСПЕРСНЫХ КАТАЛИЗАТОРОВ НА ОСНОВЕ РАЗЛИЧНЫХ МЕТАЛЛОВ
}

\author{
Соснин Глеб Андреевич ${ }^{12}$, \\ sosningleb@catalysis.ru \\ Заикина Олеся Олеговна ${ }^{1,2}$, \\ omironenko@catalysis.ru \\ Елецкий Петр Михайлович', \\ yeletsky@catalysis.ru \\ Яковлев Вадим Анатольевич ${ }^{12,}$, \\ yakovlev@catalysis.ru \\ 1 Институт Катализа имени Г.К. Борескова СО РАН, \\ Россия, 630090, г. Новосибирск, пр. Академика Лаврентьева, 5. \\ 2 Новосибирский Государственный Университет, \\ Россия, 630090, г. Новосибирск, ул. Пирогова, 2.
}

\begin{abstract}
Актуальность исследования обусловлена необходимостью разработки альтернативных водородным технологий переработки тяжелого нефтяного сырья для вовлечения последнего в структуру нефтепереработки и увеличения глубины переработки остатков (мазут, гудрон) с повышением выхода светлых дистиллятов и минимизацией выхода побочных продуктов.

Цель: исследовать процесс каталитического парового крекинга гудрона западно-сибирской нефти в присутствии дисперсных катализаторов на основе различных металлов

Объекты: каталитический паровой крекинг - термокаталитический процесс облагораживания тяжелого нефтяного сырья в присутствии воды.

Методы. Для оценки эффективности катализаторов на основе различных металлов использовали совокупность физико-химических показателей: фракционный состав жидких продуктов каталитического парового крекинга (ASTM D716991-11), содержание S, соотношение H:C (HCNS-O анализ), плотность и кинематическую вязкость (ASTM D7042) жидких продуктов.

Результаты. Установлены особенности парового каталитического крекинга гудрона при $450{ }^{\circ} \mathrm{C}$ в присутствии дисперсных катализаторов на основе различных металлов (при концентрации металла в исходной эмульсии 0,5 мас. \%). Выявлено, что применение Мо-содержащего дисперсного катализатора приводит к увеличению соотношения Н:C (до 1,56) в жидких продуктах по сравнению с паровым крекингом без катализатора (1,32), также происходит снижение содержания серы в жидких продуктах (на 0,22 мас. \%), что свидетельствует об увеличении глубины взаимодействия воды и сырья при использовании дисперсного катализатора. В случае использования дисперсного катализатора на основе железа отмечено увеличение выхода светлых фракций (29,3 мас. \%), поскольку данный катализатор характеризуется высокой активностью в процессах окислительного крекинга. Остальные дисперсные катализаторы на основе Ni, Al, и Со не превосходят Мо- и Fе-содержащие катализаторы по выходу и качеству жидких продуктов облагораживания. Вязкость продуктов облагораживания снижается по сравнению с исходным гудроном приблизительно в 90 раз.
\end{abstract}

Ключевые слова:

Каталитический паровой крекинг, тяжелое нефтяное сырье, дисперсный катализатор, сларри-реактор, молибден, железо.

\section{Введение}

В условиях постоянного развития технологий и роста потребления энергоносителей спрос на жидкие продукты с низкой температурой кипения постоянно растет. Вследствие чего вовлечение тяжелого нефтяного сырья (тяжелая нефть, битум, мазут, гудрон и др., далее - ТНС) в инфраструктуру нефтеперерабатывающих производств является необходимым для увеличения глубины переработки добываемого углеводородного сырья с получением максимально возможного количества светлых дистиллятных фракций (бензиновые и дизельные). Вакуумный остаток (гудрон) как трудноперерабатываемый продукт перегонки нефти является одним из наиболее доступных и предпочтительных к переработке типов тяжелых нефтяных высококипящих фракций. Все процессы облагора- живания нефтяного сырья можно условно разделить на процессы, основанные на снижении содержания углерода в сырье, либо насыщении его водородом [1]. В основе вышеперечисленных подходов лежит термическая обработка сырья с получением маловязкой полусинтетической нефти (ПСН). Процессы, основанные на снижении содержания углерода, являются наиболее простыми в промышленной реализации с минимальными экономическими затратами. Данный подход характеризуется невысоким выходом светлых фракций, а также значительным количеством получаемых побочных продуктов (газообразные продукты и продукты уплотнения) [2]. Для снижения выхода коксовых отложений и увеличения селективности по светлым фракциям целесообразно использовать молекулярный водород [3]. Основным недостатком ги- 
дрогенизационных процессов является их высокая стоимость и опасность, связанная с использованием высоких давлений водорода [4]. В последнее время активно развиваются процессы, совмещающие преимущества двух вышеуказанных подходов, например, термокаталитические процессы с использованием воды [5-8].

В процессах облагораживания тяжелого нефтяного сырья используются катализаторы на основе различных $3 \mathrm{~d}$-металлов (Ni, Mo, Co, W, Fe и др.) [9-12]. Данные катализаторы можно разделить на нанесенные [13], грубодисперсные [7] (>10 мкм) и дисперсные. Последние, в свою очередь, делятся по типу прекурсора на получаемые in situ водо- и нефтерастворимые катализаторы $[14,15]$ и получаемые ex situ порошковые катализаторы [12]. Ocновным преимуществом нанесенных и грубодисперсных катализаторов является простое отделение катализатора после проведения реакции облагораживания [16]. Однако в данном случае работа с тяжелым нефтяным сырьем характеризуется несколькими серьезными недостатками. $\mathrm{K}$ таковым относятся следующие протекающие процессы: блокировка пор высокомолекулярными фрагментами тяжелого нефтяного сырья, диффузионные ограничения массопереносу в ходе реакции и, как следствие, быстрая дезактивация катализатоpa [17]. К преимуществам дисперсных катализаторов можно отнести увеличение доступности высокодисперсных активных центров для крупных молекул реагентов, возможность стабилизации высоковязкими компонентами каталитической дисперсии при термообработке и способность захватывать свободные радикалы на поверхности, тем самым уменьшая реакции поликонденсации [17].

Поиск активных каталитических систем в процессах глубокой переработки гудрона является интересной и актуальной научной задачей. Для получения максимального выхода дистиллятных фракций, химических веществ с соблюдением экологических норм ТНС должно быть переработано с удалением гетероатомов и превращением тяжелых фракций в более желательные светлые углеводородные фракции, которые совместимы с современными двигателями внутреннего сгорания или подходят в качестве химических прекурсоров. В настоящий момент в литературе активно обсуждаются различные каталитические подходы к переработке гудрона. Наиболее распространенными направлениями исследования являются гидрогенизационные процессы, каталитический крекинг и процессы с использованием воды (акватермолиз, каталитический паровой крекинг (КПК) и крекинг в сверхкритической воде). В гидропроцессах в основном используются катализаторы на основе молибдена [15, 18-21], которые позволяют увеличивать выходы светлых фракций и снижать коксообразование. Ряд работ посвящен модифицированию традиционных Мо-содержащих катализаторов $[16,22,23]$. Так, в работе [22] приведены результаты исследования гидрокрекинга каменноугольно- го вакуумного остатка в присутствии мультиметаллического катализатора (на основе $\mathrm{Fe}-\mathrm{Mo}-\mathrm{Ni}$ ) в сларри-режиме в автоклавной установке при температурах $330-430{ }^{\circ} \mathrm{C}$ в течение 20 мин. Показано, что один из компонентов каталитической системы $\mathrm{Fe}_{1-\mathrm{x}} \mathrm{S}$ (пирротин) в присутствии дисперсных сульфидов молибдена и никеля является активной фазой в процессе гидрокрекинга. Мультикомпонентная сульфидная каталитическая система способствует термическому разложению каменноугольного вакуумного остатка при низкой температуре, при этом подавляется коксообразование с увеличением выхода легких углеводородов и удалением гетероатомов, что связано с синергетическим әффектом между активными фазами.

Основной особенностью каталитического крекинга является отсутствие внешних доноров водорода, в связи с этим применяемые катализаторы должны обладать высокой активностью в реакциях крекинга и низкой склонностью к коксообразованию. В работе [12] для превращения гудрона (реактор периодического действия, $410-450^{\circ} \mathrm{C}, 1-2$ ч) в качестве катализатора использовался карбид вольфрама в виде частиц микронного размера. При анализе фракционного состава получаемых продуктов установлено, что катализатор WC способствует увеличению выхода светлых фракций с уменьшением содержания вакуумного газойля и образующихся побочных продуктов. Авторы предположили, что в присутствии карбида вольфрама вклад реакций уплотнения уменьшается, а увеличение образования светлых фракций связано с ростом вклада реакций по карбокатионному механизму. Авторами работы [24] изучен процесс каталитического крекинга вакуумного остатка в автоклавной установке при $400-420{ }^{\circ} \mathrm{C}$ в течение 60 мин в присутствии образующегося in situ и коммерческого оксида алюминия, а также бурового шлама, состоящего в основном из песчаника (алюмосиликаты, оксиды железа, титана). В качестве основных результатов авторы приводят снижение выхода кокса и большую конверсию вакуумного остатка в присутствии 10 мас. \% буровых шламов, по сравнению с термическим крекингом и крекингом в присутствии оксида алюминия обоих типов. Данный факт авторы объясняют равновесием между процессами образования предшественников продуктов уплотнения и их адсорбцией на поверхности частиц катализатора с дальнейшим крекингом. На основании литературных данных можно заключить, что каталитический крекинг гудрона способствует получению жидких продуктов с улучшенным фракционным составом, однако отсутствие доноров водорода приводит к ухудшению качества продуктов (снижение соотношения $\mathrm{H}: \mathrm{C}$ ) либо показателей процесса (увеличение выхода кокса и газа), по сравнению с гидропроцессами.

Для сглаживания недостатков термического/каталитического крекинга гудрона целесообразно использовать подходы, способствующие улучшению качества получаемых нефтяных фрак- 
ций за счет процессов переноса водорода из водородсодержащих источников. Одним из наиболее доступных доноров водорода является вода [25]. Как было указано ранее, процессы с использованием воды можно разделить (по ее фазовому состоянию) на акватермолиз (субкритическая вода), паровой крекинг (перегретый пар) и крекинг в сверхкритической воде $[5,26]$. Акватермолиз проводят при сравнительно мягких условиях $\left(T<374{ }^{\circ} \mathrm{C}\right.$ и давлении насыщенных паров воды), достаточных для гидролиза C-S связей в углеводородном сырье, однако недостаточных для глубокой конверсии высококипящих фракций за счет протекания термического крекинга. Как следствие, в качестве основных результатов данных процессов авторы указывают снижение вязкости и содержания серы при незначительном изменении фракционного состава получаемых жидких продуктов [27-29]. Использование сверхкритической воды в присутствии различных катализаторов (на основе $\mathrm{CeO}_{2}[30,31]$, $\mathrm{Fe}_{2} \mathrm{O}_{3}$ [32-34], $\mathrm{Co}_{3} \mathrm{O}_{4}, \mathrm{MnO}_{2}$ [30], $\mathrm{NiMo} / \mathrm{SiO}_{2}$ [35], $\left.\mathrm{ZnO}, \mathrm{Al}_{2} \mathrm{O}_{3}[36,37]\right)$ в процессах облагораживания тяжелого нефтяного сырья характеризуется высокой эффективностью благодаря как переходу в более жесткие условия $\left(T=400-500{ }^{\circ} \mathrm{C}, P>22,1 \mathrm{MПа}\right)$, так и изменениям свойств воды при переходе в сверхкритическое состояние [38]. Данный процесс зарекомендовал себя в качестве эффективного подхода к облагораживанию тяжелого нефтяного сырья (в т. ч. гудрона) с получением облегченной качественной полусинтетической нефти с минимальным выходом кокса. Однако дороговизна данного подхода, а также необходимость использования специальных коррозионностойких сплавов для реакторов и других технологических узлов делают данный процесс нерентабельным в промышленных масштабах.

Использование воды в виде перегретого пара при $T=400-500{ }^{\circ} \mathrm{C}$ позволяет решить проблемы недостаточной эффективности облагораживания сырья в случае акватермолиза и при этом не требует значительных экономических вложений, связанных с модернизаций установок, при реализации каталитического парового крекинга на существующих НПЗ. В процессе КПК тяжелого нефтяного сырья используются катализаторы на основе $\mathrm{Mo}[39,40], \mathrm{Ni}[6,41]$ и $\mathrm{Fe}[14,42]$. В данных работах в качестве основных положительных результатов можно выделить увеличение выхода жидких продуктов с ростом в последних соотношения Н:C и снижением содержания S. Кроме того, отмечается снижение выхода кокса по сравнению с термическим крекингом без использования воды и катализатора. Однако, данное направление характеризуется отсутствием достаточной научной базы. Как следствие, для изучения всех аспектов КПК влияния природы активных фаз каталитических систем на основе различных металлов и физикохимических параметров процесса на выход и свойства получаемых продуктов, требуется дальнейшее проведение научно-исследовательских работ.
Таким образом, поиск и исследование активных каталитических систем для крекинга ТНС в присутствии водяного пара является актуальной задачей. Целью данной работы является изучение каталитической активности дисперсных катализаторов на основе ряда металлов ( $\mathrm{Mo}, \mathrm{Ni}, \mathrm{Al}, \mathrm{Co}, \mathrm{Fe})$ в процессе каталитического парового крекинга гудрона как наиболее часто используемых металлов, обладающих различными функциями, в катализаторах для переработки тяжелого нефтяного сырья.

Экспериментальная часть.

\section{Свойства используемого гудрона}

В экспериментах использовали гудрон западносибирской нефти АО «Газпромнефть-ОНПЗ» с высоким содержанием серы (1,8 мас. \%), преимущественно состоящий из углеводородных фракций с температурой кипения выше $500^{\circ} \mathrm{C}$ (табл. 2). В табл. 1 представлены данные по CHNS-составу, плотности и вязкости, а также коксуемости по Конрадсону.

Таблица 1. Основные характеристики использованного в экспериментах гудрона

Table 1. The main characteristics of the vacuum residue used in the experiments

\begin{tabular}{|c|c|c|c|}
\hline $\begin{array}{c}\text { Элементный } \\
\text { состав } \\
\text { Elemental } \\
\text { composition }\end{array}$ & \begin{tabular}{|c|} 
Содержание, \\
мac. \% \\
Content, \\
wt. \%
\end{tabular} & $\begin{array}{c}\text { Атомное соотношение } \mathrm{H}: \mathrm{C} \\
\text { Atomic ratio } \mathrm{H}: \mathrm{C}\end{array}$ & 1,57 \\
\hline C & 85,6 & $\begin{array}{c}\text { Плотность при } 25^{\circ} \mathrm{C}, \mathrm{r} / \mathrm{cm}^{3} \\
\text { Density at } 25^{\circ} \mathrm{C}, \mathrm{g} / \mathrm{cm}^{3}\end{array}$ & 1,00 \\
\hline $\mathrm{H}$ & 11,2 & $\begin{array}{c}\text { Кинематическая вязкость } \\
\text { при } 60{ }^{\circ} \mathrm{C} \text {, сCT } \\
\text { Kinematic viscosity at } 60{ }^{\circ} \mathrm{C} \text {, cSt }\end{array}$ & 6712,5 \\
\hline$N$ & 0,9 & \multirow{3}{*}{$\begin{array}{c}\text { Коксуемость } \\
\text { по Конрадсону, мас. \% } \\
\text { Conradson residue, wt. \% }\end{array}$} & \multirow{3}{*}{19,6} \\
\hline$S$ & 1,8 & & \\
\hline 0 & 0,5 & & \\
\hline
\end{tabular}

\section{Методика приготовления катализаторов}

Дисперсный катализатор на основе различных металлов формировался in situ в горячей зоне реактора из приготовленной ранее обратной эмульсии водного раствора прекурсора катализатора в гудроне. Методика приготовления обратной эмульсии заключается в равномерном диспергировании соответствующего количества водного раствора предшественника катализатора (парамолибдат аммония, сульфат алюминия, нитрат никеля, хлорид кобальта, сульфат железа II) в нагретом до $80{ }^{\circ} \mathrm{C}$ гудроне. Количество воды и прекурсора выбиралось таким образом, чтобы в состав конечной эмульсии входило 10 \% воды и 0,5 \% металла. Диспергирование осуществляли с использованием диспергатора IKA T-25 basic ULTRA-TURRAX при скорости перемешивания 24000 об/мин в течение 3 мин.

\section{Методика проведения эксперимента}

Исследование каталитического парового крекинга гудрона проводили на установке с проточным реактором типа сларри (рис. 1) при температуре $450{ }^{\circ} \mathrm{C}$ и давлении 2,0 МПа. Внутренний диа- 
метр реактора - 20 мм, длина - 300 мм, материал нержавеющая сталь.

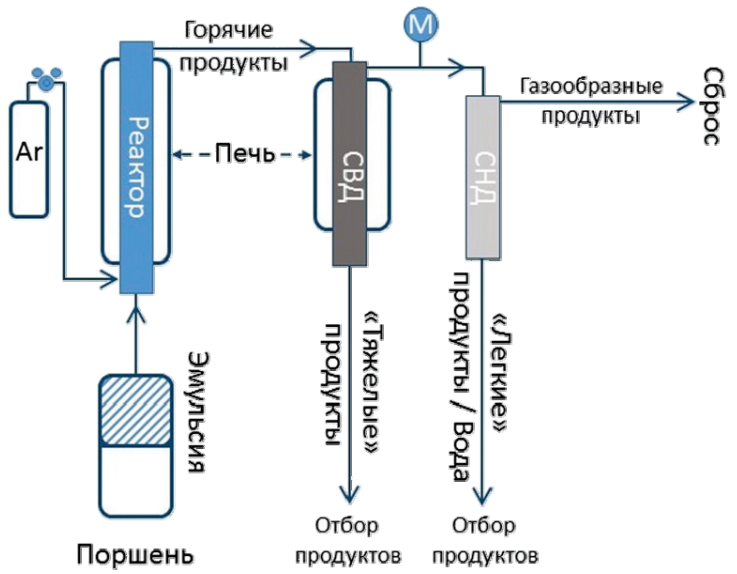

Рис. 1. Схема проточной установки с реактором типа сларри для проведения экспериментов по каталитическому паровому крекингу гудрона

Fig. 1. Scheme of a flow installation with slurry-type reactor for experiments on catalytic steam cracking of vacuum residue

Подача обратной эмульсии водного раствора предшественника в реактор обеспечивается с помощью поршня, подогреваемого до $60{ }^{\circ} \mathrm{C}$. Скорость подачи сырья составляла 100 мл/ч (в случае термического крекинга - чистого гудрона, в случае парового крекинга - обратной эмульсии воды в гудроне). В реактор также подавали аргон для достижения общего давления в системе 2,0 МПа, со скоростью 100 мл/мин. «Тяжелые» продукты накапливались в сепараторе высокого давления (СВД), нагретого до $230{ }^{\circ} \mathrm{C}$, более «легкие» продукты и вода далее поступали в сепаратор низкого давления (СНД), охлаждаемого до комнатной температуры. Жидкие продукты крекинга после отделения воды на делительной воронке объединяли и анализировали. Определение выхода продуктов уплотнения проводили путем суммирования массы остатков в реакторе, после отмывки дихлорметаном, с содержанием кокса в жидких продуктах, которое определяли следующим образом: часть жидких продуктов растворяли в избытке дихлорметана с последующим центрифугированием и промыванием остатка на бумажном фильтре.

Продукты переработки гудрона исследовали следующими физико-химическими методами:

HCNS-O анализ исходного сырья и жидких продуктов переработки гудрона

Элементный CHNS-состав жидких и твердых образцов определяли на CHNS-O анализаторе VARIO EL CUBE (Elementar Analysensysteme, Германия). Пробу анализировали не менее трех раз с последующим усреднением полученных данных.

Определение фракционного состава жидких

нефтепродуктов по ASTM D716991-1-1

Фракционный состав исходного гудрона и объединенных жидких продуктов его переработки определяли с помощью высокотемпературной газовой хроматографии в соответствии со стандартом ASTM D71691-11. В качестве стандартного вещества для определения степени извлечения использовали вакуумный газойль.

\section{Определение кинематической вязкости}

Кинематическую вязкость измеряли исходя из величин плотности и динамической вязкости, определенных на вискозиметре Штабингера по стандарту ASTM D7042. Кинематическую вязкость измеряли в диапазоне температур 20-100 ${ }^{\circ} \mathrm{C}$ в зависимости от исследуемого образца.

\section{Определение плотности}

Определение плотности исходного углеводородного сырья и продуктов переработки проводили на вискозиметре Штабингера по стандарту ASTM D7042.

\section{Результаты и обсуждение}

Для исследования влияния природы катализатора на выход и свойства продуктов термического превращения гудрона были проведены эксперименты по термическому крекингу (ТК), паровому крекингу (ПК) и каталитическому паровому крекингу (КПК) в присутствии дисперсных катализаторов на основе различных металлов. Оценку әффективности процессов проводили по совокупности параметров: выхода светлых фракций $\left(T_{\text {кип }}<350{ }^{\circ} \mathrm{C}\right)$ и полусинтетической нефти (жидких продуктов в целом), а также нефтяного кокса и газообразных продуктов (табл. 2). Также оценивались параметры, связанные с качественными характеристиками жидких продуктов: атомное соотношение Н:C , содержание серы, вязкость и плотность (табл. 3).

Использование Мо-содержащего дисперсного катализатора в процессе каталитического парового крекинга гудрона по сравнению с паровым крекингом без катализатора (ПК) позволяет при практически неизменном выходе жидких продуктов (86,5 мас. \% ) увеличить выход светлых фракций (с 20,9 до 23,0 мас. \%), снизить содержание серы на 0,22 мас. \% и увеличить соотношение $\mathrm{H}: \mathrm{C}$ на 0,24 в жидких продуктах. Также обнаружено, что его использование не приводит к увеличению выхода продуктов уплотнения (кокс), однако выход газообразных продуктов увеличивается на 0,9 мас. $\%$, что, вероятнее всего, связано с активностью Мо-содержащего дисперсного катализатора в процессе обессеривания тяжелого нефтяного сырья с образованием газообразных серосодержащих продуктов [40].

В случае никеля, по сравнению с молибденом, наблюдается незначительное повышение содержания серы в жидких продуктах (до 1,32 мас. \%) и увеличение выхода кокса $(6,1$ мас. \%), что, в свою очередь, приводит к снижению выхода жидких продуктов (на 5,5 мас. \%). Это объясняется большей склонностью никеля к коксообразованию как 
в каталитическом крекинге, так и в каталитическом паровом крекинге, что согласуется с данными, полученными в процессе каталитического парового крекинга тяжелой нефти в статическом режиме [6].

Таблица 2. Выход продуктов термического крекинга, парового крекинга гудрона с 0,5 мас. \% различных металлов при соотношении вода:гудрон=0, 1:1. Условия процесса: температура - $450{ }^{\circ} \mathrm{C}$; давление 2,0 МПа, скорость подачи гудрона - 0,1 кг/4

Table 2. Yield of thermal cracking (TC), steam cracking (SC) products of vacuum residue (VR) with 0,5 wt. \% of various metals with water:vacuum residue $=0,1: 1 \mathrm{ra}$ tio. Process conditions: temperature $-450{ }^{\circ} \mathrm{C}$; pressure 2,0 MPa, feed rate $-0,1 \mathrm{~kg} / \mathrm{h}$

\begin{tabular}{|c|c|c|c|c|c|c|c|c|}
\hline \multirow[t]{2}{*}{$\begin{array}{c}\text { Выход фракций, } \\
\text { мас. \% } \\
\text { Fraction yield, wt. \% }\end{array}$} & \multirow[t]{2}{*}{$\begin{array}{c}\text { Гудрон } \\
\text { VR }\end{array}$} & \multirow[t]{2}{*}{$\begin{array}{l}\text { TK } \\
\text { TC }\end{array}$} & \multirow[t]{2}{*}{$\begin{array}{l}\Pi \mathrm{K} \\
\mathrm{SC}\end{array}$} & \multicolumn{5}{|c|}{$\begin{array}{c}\text { Продукты крекинга на } \\
\text { различных катализаторах } \\
\text { (металл - основа диспер } \\
\text { сного катализатора) } \\
\text { Products on various } \\
\text { metals - the basis of the } \\
\text { dispersed catalysts }\end{array}$} \\
\hline & & & & Mo & $\mathrm{Ni}$ & $\mathrm{Fe}$ & $\mathrm{CO}$ & $\mathrm{Al}$ \\
\hline $\begin{array}{c}\text { Газообразные } \\
\text { продукты } \\
\text { Gas }\end{array}$ & - & $\mid$\begin{tabular}{|l}
10,1 \\
\end{tabular} & 5,5 & 6,4 & 4,9 & 8,2 & 5,8 & 5,2 \\
\hline $\begin{array}{c}\text { Бензиновая н.к. } \\
-200^{\circ} \mathrm{C} \\
\text { Gasoline b.p. } \\
-200^{\circ} \mathrm{C}\end{array}$ & 0 & 16,6 & 8,4 & 10,3 & 6,8 & 13,0 & 8,6 & |9,9 \\
\hline $\begin{array}{c}\text { Дизельная/Diesel } \\
200-360{ }^{\circ} \mathrm{C} \\
\end{array}$ & 0 & 21,6 & 12,5 & 12,7 & 9,6 & 16,3 & 12,0 & 11,9 \\
\hline $\begin{array}{c}\text { Масляная/Gasoil } \\
360-500^{\circ} \mathrm{C}\end{array}$ & 5 & 12,2 & 15,1 & 15,6 & 12,2 & 11,4 & 13,7 & 14,9 \\
\hline $\begin{array}{c}\text { Остаточные/Residue } \\
T>500^{\circ} \mathrm{C}\end{array}$ & 95 & 16,9 & 49,2 & 47,9 & 52,4 & 41,9 & 51,5 & 46,1 \\
\hline \begin{tabular}{|c|} 
Светлые н.K. \\
$-360^{\circ} \mathrm{C}$ \\
Light fractions b.p. \\
$-360^{\circ} \mathrm{C}$ \\
\end{tabular} & 0 & 38,2 & 20,9 & 23,0 & 16,4 & 29,3 & 20,6 & 21,8 \\
\hline $\begin{array}{c}\text { Выход ПСН } \\
\text { Liquid products }\end{array}$ & - & 65,8 & 85,2 & 86,5 & 81,0 & 82,6 & 85,8 & 82,8 \\
\hline Выход кокса/Coke & - & 15,5 & 3,1 & 3,0 & 6,1 & 5,5 & 3,2 & 4,7 \\
\hline
\end{tabular}

В экспериментах с использованием $\mathrm{Al}$-содержащего дисперсного катализатора не наблюдается ярко выраженной крекирующей способности, характерной для оксидных форм алюминия ввиду отсутствия значительных различий во фракционном составе жидких продуктов, по сравнению с паровым крекингом без катализатора. Мы предполагаем, что отсутствие каталитической активности данного катализатора может быть связано либо с быстрой его дезактивацией, либо с неколичественным превращением прекурсора (сульфат алюминия) в активные оксидные формы $\left(\gamma-\mathrm{Al}_{2} \mathrm{O}_{3}\right)$.

Использование $\mathrm{Fe}$-содержащих дисперсных катализаторов позволяет значительно увеличить выход светлых фракций, вероятнее всего, благодаря протеканию процесса окислительного крекинга [34]. При этом также наблюдается увеличение выхода газообразных продуктов (согласно механиз- му, за счет образования $\mathrm{CO}$ и $\mathrm{CO}_{2}$ ) и продуктов уплотнения по сравнению с КПК в присутствии Мо-содержащего катализатора. То есть возрастает конверсия гудрона в целом, что, возможно, связано локальными перегревами на поверхности катализатора ввиду экзотермичности процесса окисления. Однако Fе-содержащий дисперсный катализатор не обладает столь выраженными обессеривающими свойствами по сравнению с катализатором на основе молибдена, что приводит к увеличению содержания серы до 1,34 мас. \% в жидких продуктах облагораживания.

Таблица 3. Свойства жидких продуктов термического крекинга, парового крекинга гудрона с 0,5 мас. \% различных металлов при соотношении вода:гудрон=0,1:1. Условия процесса: температура $450^{\circ} \mathrm{C}$; давление 2,0 МПа, скорость подачи гудро$\mathrm{Ha}-0,1 \mathrm{Kr} / 4$

Table 3. Features of thermal cracking (TC), steam cracking (SC) liquid products of vacuum residue (VR) with 0,5 wt. \% of various metals with water:vacuum residue $=0,1: 1$ ratio. Process conditions: temperature$450{ }^{\circ} \mathrm{C}$; pressure 2,0 MPa, feed rate $-0,1 \mathrm{~kg} / \mathrm{h}$

\begin{tabular}{|c|c|c|c|c|c|c|c|c|}
\hline \multirow[t]{2}{*}{$\begin{array}{l}\text { Показатель } \\
\text { Parameter of liquid } \\
\text { products }\end{array}$} & \multirow[t]{2}{*}{$\mid \begin{array}{c}\text { Гудрон } \\
\text { VR }\end{array}$} & \multirow[t]{2}{*}{\begin{tabular}{|l|} 
TK \\
TC
\end{tabular}} & \multirow[t]{2}{*}{$\begin{array}{l}\Pi K \\
\text { SC }\end{array}$} & \multicolumn{5}{|c|}{\begin{tabular}{|c} 
Продукты крекинга на \\
различных катализаторах \\
(металл - основа дис- \\
персного катализатора) \\
Products on various \\
metals - the basis of the \\
dispersed catalysts
\end{tabular}} \\
\hline & & & & Mo & \begin{tabular}{|l}
$\mathrm{Ni}$ \\
\end{tabular} & $\mathrm{Fe}$ & Co & $\mathrm{Al}$ \\
\hline $\begin{array}{c}\text { Содержание S в } \\
\text { ПСН, мac. \% } \\
\text { S content, wt. \% }\end{array}$ & 1,8 & 1,63 & 1,50 & 1,28 & 1,32 & 1,34 & 1,29 & 1,33 \\
\hline $\mathrm{H}: \mathrm{C}$ & 1,57 & 1,23 & 1,32 & 1,56 & 1,57 & 1,60 & 1,57 & 1,35 \\
\hline \begin{tabular}{|c|} 
Плотность ПСН, \\
$\mathrm{k} / \mathrm{M}^{3}\left(25^{\circ} \mathrm{C}\right)$ \\
Density, $\mathrm{kg} / \mathrm{m}^{3}\left(25^{\circ} \mathrm{C}\right)$
\end{tabular} & 1000 & 890 & 935 & 940 & 955 & 915 & 950 & 937 \\
\hline $\begin{array}{c}\text { Вязкость ПСН, ССТ } \\
\left(60^{\circ} \mathrm{C}\right) \\
\text { Viscosity, } \mathrm{cSt}\left(60^{\circ} \mathrm{C}\right) \\
\end{array}$ & 6712,5 & 52 & 125 & 62 & 190 & 88 & 128 & 76 \\
\hline
\end{tabular}

В случае Со-содержащих дисперсных катализаторов наблюдаются схожие с молибденом выход и качество продуктов парового крекинга. Однако при одинаковой степени обессеривания, в случае кобальта, наблюдается меньшая конверсия гудрона в легкие фракции (выход остаточных фракций больше на 3,6 мас. \%). Это может быть связано с тем, что оксидные формы молибдена, присутствующие в системе $\left(\mathrm{MoO}_{3}\right.$ и $\left.\mathrm{MoO}_{2}\right)$, могут участвовать в окислительном крекинге наподобие оксидов железа (рис. 2).

Анализ жидких продуктов КПК показал, что процесс каталитического крекинга приводит к снижению вязкости по сравнению с исходным гудроном приблизительно в 90 раз: $62-75$ сСт и 6712,5 сСт при $60{ }^{\circ} \mathrm{C}$, соответственно. Также наблюдалось снижение плотности с 1,00 г $/ \mathrm{cm}^{3}$ (исход-

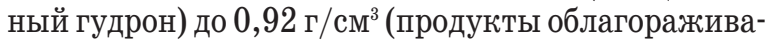
ния). Наблюдаемое снижение вязкости и плотности продуктов облагораживания гудрона объясняется 
изменением его состава в результате термокаталитической обработки в присутствии воды.

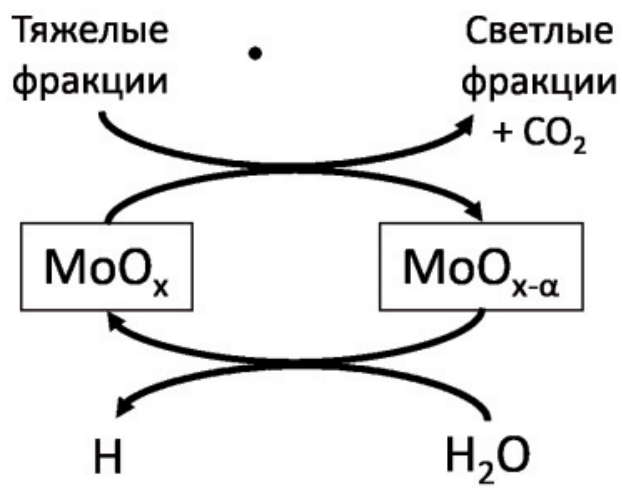

Рис. 2. Предполагаемый принцип работы Мо-содержащего дисперсного катализатора в процессе каталитического парового крекинга тяжелого нефтяного сырья

Fig. 2. Presumptive working principle of Mo-based dispersed catalysts in catalytic steam cracking of heavy oil feedstocks

Полученные результаты свидетельствуют о целесообразности использования дисперсных катализаторов на основе Мо и $\mathrm{Fe}$ в паровом крекинге тяжелого нефтяного сырья.

\section{Заключение}

Исследованы особенности парового каталитического крекинга гудрона при $450{ }^{\circ} \mathrm{C}$ в присутствии

\section{СПИСОК ЛИТЕРАТУРЫ}

1. A review of recent advances in catalytic hydrocracking of heavy residues / R. Sahu, B.J. Song, J.S. Im, Y.P. Jeon, C.W. Lee // Journal of Industrial and Engineering Chemistry. - 2015. V. 27. - P. 12-24.

2. Castañeda L.C., Muñoz J.A.D., Ancheyta J. Current situation of emerging technologies for upgrading of heavy oils // Catalysis Today. - 2014. - V. 220-222. - P. 248-273.

3. Ancheyta J., Speight J.G. Hydroprocessing of heavy oils and residua. - London: CRC Press, 2007. - 376 p.

4. Fathi M.M., Pereira-Almao P. Catalytic aquaprocessing of arab light vacuum residue via short space times // Energy and Fuels. 2011. - V. 25. - № 11. - P. 4867-4877.

5. Catalytic Steam Cracking of Heavy Oil Feedstocks: a Review P.M. Eletskii, 0.0. Mironenko, R.G. Kukushkin, G.A. Sosnin, V.A. Yakovlev // Catalysis in Industry. - 2018. - V. 10. - № 3. P. $185-201$.

6. Investigating the process of heavy crude oil steam cracking in the presence of dispersed catalysts. II: Investigating the effect of $\mathrm{Ni}$ containing catalyst concentration on the yield and properties of products / P.M. Eletskii, 0.0. Mironenko, G.A. Sosnin, 0.A. Bulavchenko, 0.A. Stonkus, V.A. Yakovlev // Catalysis in Industry. - 2016. - V. 8. - № 4. - P. 328-335.

7. Steam cracking of coal-derived liquids and some aromatic compounds in the presence of haematite / V.I. Sharypov, B.N. Kuznetsov, N.G. Beregovtsova, S.V. Baryshnikov, V.N. Sidel'nikov // Fuel. - 1996. - V. 75. - № 7. - P. 791-794.

8. Kinetic Model for Catalytic Cracking of Heavy Oil with a Zirconia-Alumina-Iron 0xide Catalyst in a Steam Atmosphere / E. Fumoto, A. Matsumura, S. Sato, T. Takanohashi // Energy and Fuels. - 2009. - V. 23. - № 11. - P. 5308-5311. дисперсных катализаторов на основе различных металлов (концентрации металлов в исходной эмульсии 0,5 мас. \%). Выявлено, что применение Мо-содержащего дисперсного катализатора приводит к увеличению соотношения $\mathrm{H}: \mathrm{C}$ (до 1,56$)$ в жидких продуктах, по сравнению с паровым крекингом без катализатора $(1,32)$. Помимо этого, происходит снижение содержания серы в жидких продуктах (на 0,22 мас. \% ), что свидетельствует об увеличении глубины взаимодействия воды и сырья при использовании дисперсного катализатора. Катализаторы на основе железа эффективны в получении светлых продуктов (выход светлых фракций 29,3 мас. \%), поскольку характеризуются высокой активностью в процессах окислительного крекинга. Остальные дисперсные катализаторы на основе $\mathrm{Ni}, \mathrm{Al}$, и $\mathrm{Co}$ не превосходят $\mathrm{Mo}^{-}$и $\mathrm{Fe}$ содержащие катализаторы по выходу и качеству жидких продуктов облагораживания. Вязкость и плотность жидких продуктов всех процессов облагораживания имеет схожие значения и падает приблизительно на 99,98 и 8 \% от исходного значения соответственно.

Работа выполнена при финансовой поддержке Министерства науки и высшего образования Российской Федераиии: соглашение № 14.607.21.0172, идентификаиионный номер соглашения RFMEFI60717X0172, название «Разработка новых технологических решений облагораживания углеводородного сырья, минимизирующих или исключаюших образование отходов и негативного воздействия на окружающую среду».

9. Highly active nonpromoted hydrotreating catalysts through the controlled growth of a supported hexagonal $\mathrm{WS}_{2}$ phase / T. Alphazan, A. Bonduelle-Skrzypczak, C. Legens, A.S. Gay, Z. Boudene, M. Girleanu, O. Ersen, C. Cop?ret, P. Raybaud // ACS Catalysis. - 2014. - V. 4. - № 12. - P. 4320-4331.

10. Catalytic steam cracking of heavy crude oil with molybdenum and nickel nanodispersed catalysts / 0.0. Mironenko, G.A. Sosnin, P.M. Eletskii, Y.K. Gulyaeva, 0.A. Bulavchenko, 0.A. Stonkus, V.0. Rodina, V.A. Yakovlev // Catalysis in Industry. - 2017. V. 9. - № 3. - P. 221-229.

11. Recovery of useful lighter fuels from petroleum residual oil by oxidative cracking with steam using iron oxide catalyst / S. Funai, E. Fumoto, T. Tago, T. Masuda // Chemical Engineering Science, - 2010, - V. 65. - № 1. - P. 60-65.

12. Каталитические свойства порошков карбида вольфрама в процессе крекинга тяжелого углеводородного сырья / М.А. Морозов, А.С. Акимов, С.П. Журавков, Н.Ю. Золотухина, Н.Н. Свириденко, А.К. Головко, А.В. Восмериков, Т.А. Федущак // Известия Томского политехнического университета. Инжиниринг георесурсов. - 2017. - V. 328. - № 8. - P. 16-24.

13. Catalytic bitumen cracking in sub- and supercritical water / X.K. Gai, H. Arano, P. Lu, J.W. Mao, Y. Yoneyama, C.X. Lu, R.Q. Yang, N. Tsubaki // Fuel Processing Technology. - 2016. V. 142. - P. 315-318.

14. Clark P.D., Kirk M.J. Studies on the Upgrading of Bituminous Oils with Water and Transition Metal Catalysts // Energy and Fuels. - 1994. - V. 8. - № 2. - P. 380-387.

15. Khadzhiev S.N., Kadiev K.M., Kadieva M.K. Synthesis and properties of nanosized systems as efficient catalysts for hydroconversion of heavy petroleum feedstock // Petroleum Chemistry. 2014. - V. 54. - № 5. - P. 323-346. 
16. Upgrading of vacuum residue in batch type reactor using Ni-Mo supported on goethite catalyst / R. Sahu, B.J. Song, Y.P. Jeon, C.W. Lee // Journal of Industrial and Engineering Chemistry. 2016. - V. 35. - P. 115-122.

17. A review of experimental procedures for heavy oil hydrocracking with dispersed catalyst / M.J. Angeles, C. Leyva, J. Ancheyta, S. Ramírez // Catalysis Today. - 2014. - V. 220-222. - P. 274-294.

18. Comparative reactivity between thermal and catalytic hydrocracking of vacuum residue: Effect of asphaltenes / N.T. Nguyen, S. Park, J. Jung, J. Cho, C.W. Lee, Y.K. Park // Journal of Industrial and Engineering Chemistry. - 2018. - V. 61. - P. 32-38.

19. A comparative activity study of a new ultra-dispersed catalyst system for a hydrocracking/hydrotreating technology using vacuum residue oil: Merey/Mesa / G. Noguera, S. Araujo, J. Hernández, A. Rivas, D. Mendoza, 0. Castellano // Chemical Engineering Research and Design. - 2012. - V. 90. - № 11. P. 1979-1988.

20. S.H. Lim, K.S. Go, N.S. Nho, J.G. Lee / Effect of reaction temperature and time on the products and asphaltene dispersion stability in slurry-phase hydrocracking of vacuum residue // Fuel. 2018 - V. 234. - P. 305-311.

21. K.S. Go, S.H. Lim, Y.K. Kim, E.H. Kwon, N.S. Nh / Characteristics of slurry-phase hydrocracking for vacuum residue with reaction temperature and concentrations of $\mathrm{MoS}_{2}$ dispersed catalysts // Catalysis Today. - 2018. - V. 305, - P. 92-101.

22. Multi-metal catalysts for slurry-phase hydrocracking of coal-tar vacuum residue: Impact of inherent inorganic minerals / J. Du, W. Deng, C. Li, Z. Zhang, T. Yang, X. Cao, F. Du, Q. Sun // Fuel. - 2018. - V. 215. - P. 370-377.

23. Manek E., Haydary J. Hydrocracking of vacuum residue with solid and dispersed phase catalyst: Modeling of sediment formation and hydrodesulfurization // Fuel Processing Technology. 2017. - V. 159. - P. 320-327.

24. Eshraghian A., Husein M.M. Catalytic thermal cracking of Athabasca VR in a closed reactor system // Fuel. - 2018. - V. 217. P. 409-419.

25. Dutta R.P., McCaffrey W.C., Gray M.R., Muehlenbachs K. Ther mal Cracking of Athabasca Bitumen: Influence of Steam on Reaction Chemistry // Energy \& Fuels. - 2000. - V. 14. - № 3. P. 671-676.

26. Heavy Oil Upgrading in the Presence of Water / P.M. Eletskii, G.A. Sosnin, 0.0. Zaikina, R.G. Kukushkin, V. Yakovlev // Journal of Siberian Federal University. Chemistry. - 2017. - V. 10. № 4. - P. 545-572.

27. Kapadia P.R., Kallos M.S., Gates I.D. A review of pyrolysis, aquathermolysis, and oxidation of Athabasca bitumen // Fuel Processing Technology. - 2015. - V. 131. - P. 270-289.

28. Mechanism of catalytic aquathermolysis: Influences on heavy oil by two types of efficient catalytic ions: $\mathrm{Fe}^{3+}$ and $\mathrm{M}^{6+} / \mathrm{Y}$. Wang, Y. Chen, J. He, P. Li, C. Yang // Energy and Fuels. - 2010. V. 24. - № 3. - P. 1502-1510.

29. Upgrading and visbreaking of super-heavy oil by catalytic aquathermolysis with aromatic sulfonic copper / K. Chao, Y. Chen, J. Li, X. Zhang, B. Dong // Fuel Processing Technology. 2012. - V. 104. - P. 174-180.
30. Golmohammadi M., Ahmadi S.J., Towfighi J. Catalytic cracking of heavy petroleum residue in supercritical water: Study on the effect of different metal oxide nanoparticles // The Journal of Supercritical Fluids. - 2016. - V. 113. - P. 136-143.

31. Catalytic cracking reaction of heavy oil in the presence of cerium oxide nanoparticles in supercritical water / M. Dejhosseini, T. Aida, M. Watanabe, . Takami, D. Hojo, N. Aoki, T. Arita, A. Kishita, T. Adschiri // Energy and Fuels. - 2013. - V. 27. - № 8. P. 4624-4631.

32. Hosseinpour M., Ahmadi S.J., Fatemi S. Successive co-operation of supercritical water and silica-supported iron oxide nanoparticles in upgrading of heavy petroleum residue: Suppression of coke deposition over catalyst // Journal of Supercritical Fluids. 2015. - V. 100. - P. 70-78.

33. Hosseinpour M., Fatemi S., Ahmadi S.J. Catalytic cracking of petroleum vacuum residue in supercritical water media: Impact of $\alpha-\mathrm{Fe}_{2} \mathrm{O}_{3}$ in the form of free nanoparticles and silica-supported granules // Fuel. - 2015. - V. 159. - P. 538-549.

34. Fumoto E., Tago T., Masuda T. Production of lighter fuels by cracking petroleum residual oils with steam over zirconia-supporting iron oxide catalysts // Energy and Fuels. - 2006. - V. 20. № 1. - P. 1-6.

35. Anthracene aquacracking using $\mathrm{NiMo} / \mathrm{SiO}_{2}$ catalysts in supercritical water conditions / T.R. Reina, P. Yeletsky, J.M. Bermúdez, P. Arcelus-Arrillaga, V.A. Yakovlev, M. Millan // Fuel. - 2016. - V. 182. - P. 740-748.

36. Fedyaeva 0.N., Vostrikov A.A. Hydrogenation of bitumen in situ in supercritical water flow with and without addition of zinc and aluminum // Journal of Supercritical Fluids. - 2012. - V. 72. P. $100-110$.

37. Fedyaeva 0.N., Antipenko V.R., Vostrikov A.A. Conversion of sulfur-rich asphaltite in supercritical water and effect of metal additives // Journal of Supercritical Fluids. - 2014. - V. 88. P. 105-116.

38. Weingärtner H., Franck E.U. Supercritical water as a solvent // Angewandte Chemie - International Edition. - 2005. - V. 44. № 18. - P. 2672-2692.

39. Vacuum residue upgrading through hydroprocessing with subcritical water / H.K. Ahn, S.H. Park, S. Sattar, S.I. Woo // Catalysis Today. - 2016. - V. 265. - P. 118-123.

40. A study of the catalytic steam cracking of heavy crude oil in the presence of a dispersed molybdenum-containing catalyst / 0.0. Mironenko, G.A. Sosnin, P.M. Eletskii, Y.K. Gulyaeva, 0.A. Bulavchenko, 0.A. Stonkus, V.0. Rodina, V.A. Yakovlev // Petroleum Chemistry. - 2017. - V. 57. - № 7. - P. 618-629.

41. Cabrales-Navarro F.A., Pereira-Almao P. Catalytic Steam Cracking of a Deasphalted Vacuum Residue Using a Ni/K Ultradispersed Catalyst // Energy and Fuels. - 2017. - V. 31. - № 3. P. 3121-3131.

42. Исследование процесса парового крекинга тяжелой нефти в присутствии железо- и молибденсодержащих дисперсных катализаторов в реакторе проточного типа / Р.Г. Кукушкин, П.М. Елецкий, 0.0. Заикина, Г.А. Соснин, О.А. Булавченко, В.А. Яковлев // Катализ в промышленности. - 2018. - V. 18. № 3. - P. 62-71. 


\section{Информация об авторах}

Соснин $\Gamma$.А., младший научный сотрудник лаборатории каталитических процессов переработки возобновляемого сырья Института Катализа имени Г.К. Борескова СО РАН; аспирант кафедры катализа и адсорбции, ассистент кафедры физической химии Новосибирского Государственного Университета.

Заикина O.O., кандидат химических наук, научный сотрудник лаборатории каталитических процессов переработки возобновляемого сырья Института Катализа имени Г.К. Борескова СО РАН; научный сотрудник Новосибирского Государственного Университета.

Eлеикий П.М., кандидат химических наук, научный сотрудник лаборатории каталитических процессов переработки возобновляемого сырья Института Катализа имени Г.К. Борескова СО РАН, Россия.

Яковлев В.А., доктор химических наук, заведующий лабораторией каталитических процессов переработки возобновляемого сырья Института Катализа имени Г.К. Борескова СО РАН; ведущий научный сотрудник Новосибирского Государственного Университета. 
UDC 665.658.6

\title{
CATALYTIC STEAM CRACKING OF VACUUM RESIDUE IN PRESENCE OF DISPERSED CATALYSTS BASED ON MO, NI, FE, CO, AL METALS
}

\author{
Gleb A. Sosnin ${ }^{1,2}$, \\ sosningleb@catalysis.ru \\ Olesya O. Zaikina ${ }^{12,}$ \\ omironenko@catalysis.ru \\ Petr M. Eletskii', \\ yeletsky@catalysis.ru \\ Vadim A. Yakovlev, \\ yakovlev@catalysis.ru \\ 1 Boreskov Institute of Catalysis SB RAS, \\ 5, Lavrentiev avenue, Novosibirsk, 630090, Russia. \\ ${ }^{2}$ Novosibirsk State University, \\ 2, Pirogov street, Novosibirsk, 630090, Russia.
}

The relevance of the research is caused by the need in developing alternative hydrogen-free technologies for processing heavy oil feedstocks to involve the latter in oil refining and increase the depth of processing of residues (atmospheric and vacuum residues) to minimize yield of by-products.

The main aim of the research is to investigate catalytic steam cracking of vacuum residue of West-Siberian oil in the presence of dispersed catalysts based on various metals.

Objects: catalytic steam cracking - thermocatalytic process of heavy oil feedstock upgrading in the presence of water.

Methods. To assess the efficacy of catalysts based on various metals, a complex of physicochemical parameters: fractional composition of catalytic steam cracking liquid products (ASTM D7169-11), S content, H:C ratio (HCNS-O analysis), density and kinematic viscosity (ASTM D7042) of liquid products, was used.

Results. The authors have determined the main features of steam catalytic cracking of vacuum residue at $450{ }^{\circ} \mathrm{C}$ in the presence of dispersed catalysts based on various metals (at a metal concentration in the initial emulsion of 0,5 wt. \%). It was revealed that the use of a Mo-based dispersed catalyst leads to an increase of the $\mathrm{H:C}$ ratio $($ to 1,56$)$ in liquid products comparing to steam cracking without a catalyst (1,32), also the sulfur content in liquid products decreases (by 0,22 wt. \%), these facts indicate the enhancement of interaction between water and heavy feedstocks using this type of dispersed catalyst. The use of a dispersed catalyst based on iron, an increase in the yield of light fractions $(29,3$ wt. \%) was noted, since this catalyst is characterized by high activity in oxidative cracking. The remaining dispersed catalysts based on $\mathrm{Ni}$, Al, and Co do not exceed $\mathrm{Mo}$ - and Fe-based catalysts in the yield and quality of liquid products. The viscosity of liquid products is reduced by about 90 times compared with the original vacuum residue.

\section{Key words:}

Catalytic steam cracking, heavy oil feedstocks, dispersed catalyst, slurry-reactor, molybdenum, iron.

The work is supported by the Ministry of Science and Higher Education of the Russian Federation: project no. 14.607.21.0172, identification number RFMEFI60717X0172.

\section{REFERENCES}

1. Sahu R. A review of recent advances in catalytic hydrocracking of heavy residues. Journal of Industrial and Engineering Chemistry, 2015, vol. 27, pp. 12-24.

2. Castañeda L.C., Muñoz J.A.D., Ancheyta J. Current situation of emerging technologies for upgrading of heavy oils. Catalysis Today, 2014, vol. 220-222, pp. 248-273.

3. Ancheyta J., Speight J.G. Hydroprocessing of heavy oils and residua. London, CRC Press, 2007. 376 p.

4. Fathi M.M., Pereira-Almao P. Catalytic aquaprocessing of arab light vacuum residue via short space times. Energy and Fuels, 2011, vol. 25, no. 11, pp. 4867-4877.

5. Eletskii P.M. Catalytic Steam Cracking of Heavy Oil Feedstocks: a Review. Catalysis in Industry, 2018, vol. 10, no. 3, pp. 185-201.

6. Eletskii P.M. Investigating the process of heavy crude oil steam cracking in the presence of dispersed catalysts. II: Investigating the effect of Ni-containing catalyst concentration on the yield and properties of products. Catalysis in Industry, 2016, vol. 8, no. 4, pp. 328-335.
7. Sharypov V.I. Steam cracking of coal-derived liquids and some aromatic compounds in the presence of haematite. Fuel, 1996, vol. 75, no. 7, pp. 791-794.

8. Fumoto E. Kinetic Model for Catalytic Cracking of Heavy Oil with a Zirconia-Alumina-Iron 0xide Catalyst in a Steam Atmosphere. Energy and Fuels, 2009, vol. 23, no. 11, pp. 5308-5311.

9. Alphazan T. Highly active nonpromoted hydrotreating catalysts through the controlled growth of a supported hexagonal $\mathrm{WS}_{2}$ phase. ACS Catalysis, 2014, vol. 4, no. 12, pp. 4320-4331.

10. Mironenko 0.0. Catalytic steam cracking of heavy crude oil with molybdenum and nickel nanodispersed catalysts. Catalysis in Industry, 2017, vol. 9, no. 3, pp. 221-229.

11. Funai S. Recovery of useful lighter fuels from petroleum residual oil by oxidative cracking with steam using iron oxide catalyst. Chemical Engineering Science, 2010, vol. 65, no. 1, pp. 60-65.

12. Morozov M.A., Akimov A.S., Zhuravkov S.P., Zolotukhina N.Yu., Sviridenko N.N., Golovko A.K., Vosmerikov A.V., Fedushchak T.A. Catalytic properties of tungsten carbide powders in cracking heavy petroleum feedstock. Bulletin of the Tomsk Po- 
lytechnic University. Geo Assets Engineering, 2017, vol. 328, no. 8, pp. 16-24.

13. Gai X.K. Catalytic bitumen cracking in sub- and supercritical water. Fuel Processing Technology, 2016, vol. 142, pp. 315-318.

14. Clark P.D., Kirk M.J. Studies on the Upgrading of Bituminous Oils with Water and Transition Metal Catalysts. Energy and Fuels, 1994, vol. 8, no. 2, pp. 380-387.

15. Khadzhiev S.N., Kadiev K.M., Kadieva M.K. Synthesis and properties of nanosized systems as efficient catalysts for hydroconversion of heavy petroleum feedstock. Petroleum Chemistry, 2014, vol. 54, no. 5, pp. 323-346.

16. Sahu R. Upgrading of vacuum residue in batch type reactor using $\mathrm{Ni}-\mathrm{Mo}$ supported on goethite catalyst. Journal of Industrial and Engineering Chemistry, 2016, vol. 35, pp. 115-122.

17. Angeles M.J. A review of experimental procedures for heavy oil hydrocracking with dispersed catalyst. Catalysis Today, 2014, vol. 220-222, pp. 274-294.

18. Nguyen N.T. Comparative reactivity between thermal and catalytic hydrocracking of vacuum residue: Effect of asphaltenes. Journal of Industrial and Engineering Chemistry, 2018, vol. 61, pp. 32-38.

19. Noguera G. A comparative activity study of a new ultra-dispersed catalyst system for a hydrocracking/hydrotreating technology using vacuum residue oil: Merey/Mesa. Chemical Engineering Research and Design, 2012, vol. 90, no. 11, pp. 1979-1988.

20. Lim S.H. Effect of reaction temperature and time on the products and asphaltene dispersion stability in slurry-phase hydrocracking of vacuum residue. Fuel, 2018 March, vol. 234, pp. 305-311.

21. Go K.S. Characteristics of slurry-phase hydrocracking for vacuum residue with reaction temperature and concentrations of $\mathrm{MoS}_{2}$ dispersed catalysts. Catalysis Today, 2018, vol. 305, pp. 92-101.

22. Du J. Multi-metal catalysts for slurry-phase hydrocracking of coal-tar vacuum residue: Impact of inherent inorganic minerals. $\mathrm{Fu}$ el, 2018, vol. 215, pp. 370-377.

23. Manek E., Haydary J. Hydrocracking of vacuum residue with solid and dispersed phase catalyst: Modeling of sediment formation and hydrodesulfurization. Fuel Processing Technology, 2017, vol. 159 , pp. 320-327.

24. Eshraghian A., Husein M.M. Catalytic thermal cracking of Athabasca VR in a closed reactor system. Fuel, 2018, vol. 217, pp. 409-419.

25. Dutta R.P. Thermal Cracking of Athabasca Bitumen: Influence of Steam on Reaction Chemistry. Energy \& Fuels, 2000, vol. 14, no. 3, pp. 671-676.

26. Eletskii P.M. Heavy Oil Upgrading in the Presence of Water. Journal of Siberian Federal University. Chemistry, 2017, vol. 10, no. 4, pp. 545-572.

27. Kapadia P.R., Kallos M.S., Gates I.D. A review of pyrolysis, aquathermolysis, and oxidation of Athabasca bitumen. Fuel Processing Technology, 2015, vol. 131, pp. 270-289.

28. Wang Y. Mechanism of catalytic aquathermolysis: Influences on heavy oil by two types of efficient catalytic ions: $\mathrm{Fe}^{3+}$ and $\mathrm{M}^{6+}$. Energy and Fuels, 2010, vol. 24, no. 3, pp. 1502-1510.
29. Chao K. Upgrading and visbreaking of super-heavy oil by catalytic aquathermolysis with aromatic sulfonic copper. Fuel Processing Technology, 2012, vol. 104, pp. 174-180.

30. Golmohammadi M., Ahmadi S.J., Towfighi J. Catalytic cracking of heavy petroleum residue in supercritical water: Study on the effect of different metal oxide nanoparticles. The Journal of Supercritical Fluids, 2016, vol. 113, pp. 136-143.

31. Dejhosseini M. Catalytic cracking reaction of heavy oil in the presence of cerium oxide nanoparticles in supercritical water. Energy and Fuels, 2013, vol. 27, no. 8, pp. 4624-4631.

32. Hosseinpour M., Ahmadi S.J., Fatemi S. Successive co-operation of supercritical water and silica-supported iron oxide nanoparticles in upgrading of heavy petroleum residue: Suppression of coke deposition over catalyst. Journal of Supercritical Fluids, 2015, vol. 100 , pp. 70-78.

33. Hosseinpour M., Fatemi S., Ahmadi S.J. Catalytic cracking of petroleum vacuum residue in supercritical water media: Impact of $\alpha-\mathrm{Fe}_{2} \mathrm{O}_{3}$ in the form of free nanoparticles and silica-supported granules. Fuel, 2015, vol. 159, pp. 538-549.

34. Fumoto E., Tago T., Masuda T. Production of lighter fuels by cracking petroleum residual oils with steam over zirconia-supporting iron oxide catalysts. Energy and Fuels, 2006, vol. 20, no. 1, pp. $1-6$.

35. Reina T.R. Anthracene aquacracking using $\mathrm{NiMo} / \mathrm{SiO}_{2}$ catalysts in supercritical water conditions. Fuel, 2016, vol. 182, pp. $740-748$.

36. Fedyaeva 0.N., Vostrikov A.A. Hydrogenation of bitumen in situ in supercritical water flow with and without addition of zinc and aluminum. Journal of Supercritical Fluids, 2012, vol. 72, pp. $100-110$.

37. Fedyaeva O.N., Antipenko V.R., Vostrikov A.A. Conversion of sulfur-rich asphaltite in supercritical water and effect of metal additives. Journal of Supercritical Fluids, 2014, vol. 88, pp. 105-116.

38. Weingärtner H., Franck E.U. Supercritical water as a solvent. Angewandte Chemie - International Edition, 2005, vol. 44, no. 18, pp. 2672-2692.

39. Ahn H.K. Vacuum residue upgrading through hydroprocessing with subcritical water. Catalysis Today, 2016, vol. 265, pp. 118-123.

40. Mironenko 0.0. A study of the catalytic steam cracking of heavy crude oil in the presence of a dispersed molybdenum-containing catalyst. Petroleum Chemistry, 2017, vol. 57, no. 7, pp. 618-629.

41. Cabrales-Navarro F.A., Pereira-Almao P. Catalytic Steam Cracking of a Deasphalted Vacuum Residue Using a Ni/K Ultradispersed Catalyst. Energy and Fuels, 2017, vol. 31, no. 3, pp. 3121-3131.

42. Kukushkin R.G., Eletskiy P.M., Zaikina 0.0., Sosnin G.A., Bulavchenko 0.A., Yakovlev V.A. Studies of Steam Cracking of Heavy Oil in the Presence of Iron- and Molybdenum-Containing Disperse Catalysts in a Flow Type Reactor. Kataliz v promyshlennosti, 2018, vol. 18, no. 3, pp. 54-63. In Rus.

Received: 12 November 2018.

\section{Information about the authors}

Gleb A. Sosnin, junior researcher, Boreskov Institute of Catalysis SB RAS; post-graduate student, assistant, Novosibirsk State University.

Olesya O. Zaikina, Cand. Sc, researcher, Boreskov Institute of Catalysis SB RAS; researcher, Novosibirsk State University.

Petr M. Eletskii, Cand. Sc, researcher, Boreskov Institute of Catalysis SB RAS.

Vadim A. Yakovlev, Dr. Sc, head of laboratory, Boreskov Institute of Catalysis SB RAS; leading researcher, Novosibirsk State University. 